\title{
Carrier localization in InN/InGaN multiple-quantum wells with high In-content
}

\author{
S. Valdueza-Felip, ${ }^{1,2, a)}$ L. Rigutti, ${ }^{3}$ F. B. Naranjo, ${ }^{1}$ P. Ruterana, ${ }^{4}$ J. Mangeney, ${ }^{3}$ F. H. Julien, ${ }^{3}$ \\ M. González-Herráez, ${ }^{1}$ and E. Monroy ${ }^{2}$ \\ ${ }^{1}$ Electronics Department, University of Alcalá, Madrid-Barcelona Road, km 33.6, 28871 Alcalá de Henares, \\ Spain \\ ${ }^{2}$ CEA Grenoble, INAC/SP2M, 25 Rue des Martyrs 38042, Grenoble Cedex 9, France \\ ${ }^{3}$ Institut d'Electronique Fondamentale, University of Paris Sud XI, UMR 8622 CNRS, 91405 Orsay, France \\ ${ }^{4}$ Centre de recherche sur les Ions les Matériaux et la Photonique (CIMAP), UMR 6252, CNRS, ENSICAEN, \\ CEA, UCBN, 6 Boulevard Maréchal Juin, 14050 Caen Cedex 4, France
}

(Received 27 December 2011; accepted 19 July 2012; published online 10 August 2012)

\begin{abstract}
We study the carrier localization in $\mathrm{InN} / \mathrm{In}_{0.9} \mathrm{Ga}_{0.1} \mathrm{~N}$ multiple-quantum-wells (MQWs) and bulk $\mathrm{InN}$ by means of temperature-dependent photoluminescence and pump-probe measurements at $1.55 \mu \mathrm{m}$. The S-shaped thermal evolution of the emission energy of the $\mathrm{InN}$ film is attributed to carrier localization at structural defects with an average localization energy of $\sim 12 \mathrm{meV}$. Carrier localization is enhanced in the MQWs due to well/barrier thickness and ternary alloy composition fluctuations, leading to a localization energy above $35 \mathrm{meV}$ and longer carrier relaxation time. As a result, the luminescence efficiency in the MQWs is improved by a factor of five over bulk InN. (C) 2012 American Institute of Physics. [http://dx.doi.org/10.1063/1.4742157]
\end{abstract}

The extension of the operation wavelength of III-nitrides to the near-infrared (NIR) range is possible thanks to the growth of high-quality InN films displaying a bandgap energy close to $0.65 \mathrm{eV}(1.9 \mu \mathrm{m})$ at room temperature (RT). The particular optical and electronic properties of this semiconductor have rendered it a subject of extensive research aimed at the development of devices for photovoltaics, optoelectronics (laser diodes and detectors), highspeed electronics, opto-chemical sensing, and terahertz applications. ${ }^{1}$ Furthermore, thanks to the close-to-resonant behavior at $1.5 \mu \mathrm{m}$ and saturable absorption of $\mathrm{InN}$ with recovery times in the picosecond range, ${ }^{2}$ this semiconductor has emerged as a promising choice for all-optical signal processing applications in optical communication networks.

From this point of view, low-dimensional InN-based structures such as multi-quantum-wells (MQWs) $)^{3-5}$ or quantum dots $(\mathrm{QDs})^{6,7}$ can improve the efficiency of NIR devices by tuning their operation wavelength through bandgap engineering. Furthermore, the improved carrier confinement should lead to an enhanced linear and nonlinear optical response at resonant wavelengths. In this sense, strong absorption saturation has been recently demonstrated in high-In content InN/InGaN MQWs at $1.55 \mu \mathrm{m} .{ }^{8}$ In this paper, we investigate the carrier localization in $\mathrm{InN} / \mathrm{InGaN}$ MQW structures by means of temperature-dependent photoluminescence $(\mathrm{PL})$ measurements and pump-probe spectroscopy at $1.55 \mu \mathrm{m}$. Our results show that a fivefold increase in luminescence efficiency can be obtained in these nanostructures compared to bulk InN. This is consistent with the observation of a longer carrier lifetime in the MQW structure over the bulk material.

The samples under study were grown by plasma-assisted molecular-beam epitaxy (PAMBE) on 10- $\mu$ m-thick GaN-onsapphire templates. The substrate temperature during growth was $450^{\circ} \mathrm{C}$ and the $\mathrm{N}$-limited growth rate was fixed at

\footnotetext{
${ }^{\text {a)} E-m a i l: ~ s i r o n a . v a l d u e z a-f e l i p @ c e a . f r . ~}$
}

$280 \mathrm{~nm} / \mathrm{h}$. The MQW structure, designed to have a quasiresonant interband transition at $1.55 \mu \mathrm{m}$, consists of 41 periods of $\operatorname{InN} / \mathrm{In}_{0.9} \mathrm{Ga}_{0.1} \mathrm{~N}$ QWs with well and barrier thickness of $4.5 \mathrm{~nm}$ and $7 \mathrm{~nm}$, respectively. The growth of the MQW structure starts with an InN layer $(4.5 \mathrm{~nm})$ at the substrate/ MQW interface, which assures the relaxation of at least $90 \%$ of the lattice mismatch at the first interface, as verified in situ by reflective high-energy electron diffraction and ex situ by x-ray diffraction. ${ }^{9}$ A reference sample consisting of a $1-\mu \mathrm{m}$ thick $\mathrm{InN}$ layer was grown for comparison. The InN film was deposited on top of a buffer layer consisting of 10 periods of $\mathrm{InN} / \mathrm{In}_{0.7} \mathrm{Ga}_{0.3} \mathrm{~N}(4.5 \mathrm{~nm} / 7 \mathrm{~nm})$. Atomic force microscopy images $\left(2 \times 2 \mu \mathrm{m}^{2}\right.$ scanning area) display flat surfaces with atomic terraces leading to a root-mean-square roughness $\sim 0.5 \mathrm{~nm}$ for both $\mathrm{InN}$ and MQW samples. ${ }^{10}$

A fine structural analysis of the samples was carried out by transmission electron microscopy (TEM). The TEM analysis of the InN sample shows that the InN/In ${ }_{0.7} \mathrm{Ga}_{0.3} \mathrm{~N}$ buffer layer helps to reduce the threading dislocations, which are mostly of edge and mixed type [Fig. 1(a)]. The fluctuations
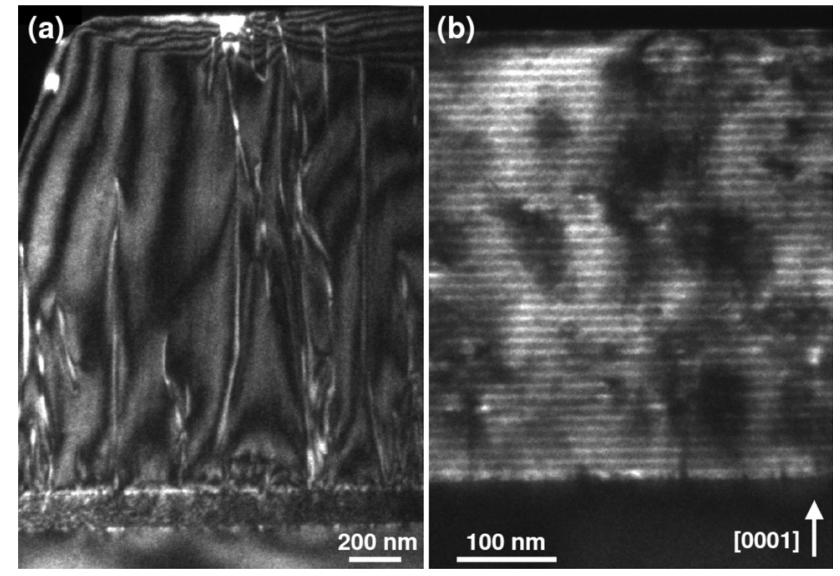

FIG. 1. Dark field $(\mathrm{g}=0002)$ TEM micrograph of (a) the $1-\mu \mathrm{m}$-thick $\mathrm{InN}$ reference sample and (b) the $\mathrm{InN} / \mathrm{In}_{0.9} \mathrm{Ga}_{0.1} \mathrm{~N}$ MQW. 
in strain induced by the QWs probably force many dislocations to bend, forming segments of misfit dislocations at the interfaces. Moreover, they may react and annihilate when bending towards or backwards the growth direction. Indeed, no other types of defects such as partial dislocations or stacking faults were seen in the layers ${ }^{11}$ and no ordering was observed to take place in the InGaN layers. As shown in Fig. 1(b), the investigated $\mathrm{InN} / \mathrm{In}_{0.9} \mathrm{Ga}_{0.1} \mathrm{~N}$ MQW sample is homogeneous, with the InN QWs exhibiting lighter contrast in this dark field image.

The optical properties were first investigated by temperature-dependent PL measurements. PL was excited by a continuous-wave $\mathrm{Ar}^{+}$laser $(\lambda=488 \mathrm{~nm})$ focused onto a $3 \mu \mathrm{m}$ diameter spot, and the emission was collected into a spectrometer equipped with an InAs detector. The lowtemperature $(T=5 \mathrm{~K}) \mathrm{PL}$ spectra of the $\mathrm{InN}$ and MQW samples are presented in the inset of Fig. 2(a). In both samples, the PL emission remains in the NIR range peaking at $\sim 1.7 \mu \mathrm{m}(0.72-0.73 \mathrm{eV})$ with a similar linewidth (full width at half maximum: FWHM $\sim 84-86 \mathrm{meV}$ ). The evolution of the normalized integrated PL intensity as a function of temperature is presented in Fig. 2(a). The PL intensity of the MQW structure drops approximately by a factor of 3 from $T=5 \mathrm{~K}$ to RT, whereas it drops by a factor of 30 for the $\mathrm{InN}$ sample. From these values, a luminescence efficiency at RT (defined as the ratio between the PL intensity at RT and at low temperature) of $\sim 16 \%$ and $\sim 3 \%$ is obtained for the MQW and InN samples, respectively. The thermal stability of the luminescence is thus strongly improved by the lowdimensional carrier confinement, as already reported in $\mathrm{InN} /$ $\mathrm{GaN}$ and $\mathrm{InGaN} / \mathrm{GaN}$ QD and QW structures. ${ }^{6,12}$

The thermal evolution of the PL emission is characterized by the intensity quenching due to the activation of nonradiative recombination processes, and also by the energy shift and broadening of the PL. For the analyzed samples, the measured thermal extinction of the PL intensity, $I(T)$, can
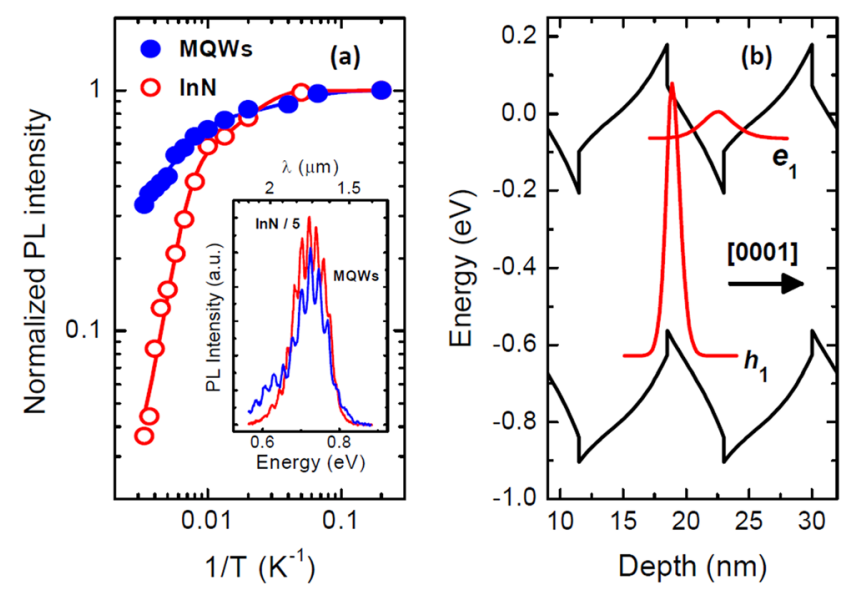

FIG. 2. (a) Thermal evolution of the integrated PL emission of the analyzed InN and MQW samples emitting at $\sim 1.7 \mu \mathrm{m}$. Solid lines are fits to Eq. (1). Inset: Low temperature $(T=5 \mathrm{~K}) \mathrm{PL}$ spectra of both samples. The superimposed oscillations are due to Fabry-Perot interference associated to the nitride epilayer thickness. It is worth saying that the thickness of the InN sample is much higher than the MQWs. (b) Band diagram of the InN/ $\mathrm{In}_{0.9} \mathrm{Ga}_{0.1} \mathrm{~N}$ MQW calculated using the nextnano3 effective-mass Schrödinger-Poisson solver, assuming a residual doping level of $10^{19} \mathrm{~cm}^{-3}$.
TABLE I. Activation and localization energies estimated for both $\mathrm{InN}$ and MQW samples.

\begin{tabular}{lccc}
\hline \hline & \multicolumn{2}{c}{$I_{P L}$ vs. $T$} & \\
\cline { 2 - 3 } Sample & $E_{a l}(\mathrm{meV})$ & $E_{a 2}(\mathrm{meV})$ & \\
\hline InN & $8 \pm 3$ & $72 \pm 9$ & $E_{l o c}(\mathrm{meV})$ \\
MQWs & $2.9 \pm 0.7$ & $36 \pm 4$ & $12 \pm 2$ \\
\hline \hline
\end{tabular}

be reproduced considering two nonradiative recombination channels: ${ }^{13}$

$$
I(T)=\frac{I(T=0 \mathrm{~K})}{1+a_{1} e^{-E_{a 1} / k_{B} T}+a_{2} e^{-E_{a 2} / k_{B} T}},
$$

where $E_{a 1}$ and $E_{a 2}$ are the activation energies of the processes related with the thermal quenching, $k_{B} T$ is the thermal energy, and $a_{1}$ and $a_{2}$ are fitting constants. The agreement of experimental data to Eq. (1) is illustrated in Fig. 2(a), and Table I summarizes the activation energies obtained for the InN and MQW samples.

To go deeper in the interpretation of these activation energies, the evolution of the PL emission energy with the temperature is compared to the Varshni equation for $\mathrm{InN}$ as illustrated in Fig. 3(a). Regarding the InN sample, the lowest activation energy $E_{a 1}=8 \pm 3 \mathrm{meV}$ obtained from the integrated intensity of the PL is in good agreement with the carrier localization energy $E_{l o c} \sim 12 \pm 2 \mathrm{meV}$ (reported in Table I) estimated from the difference between the projected PL emission energy at low temperature using the Varshni equation and the experimental value. This localization is associated with defect-related potential fluctuations, as observed by other authors. ${ }^{6,14}$ Furthermore, the localization energy corresponds to the expected value in a sample with a residual carrier concentration in the range of $n_{0} \approx 10^{19} \mathrm{~cm}^{-3} .{ }^{14}$

Regarding the MQW sample, the evolution of the PL peak energy with temperature presents a strong deviation from the expected one in bulk InN described by the Varshni equation, as shown in Fig. 3(b). The observed blue shift of the MQWs emission peak energy compared to Varshni
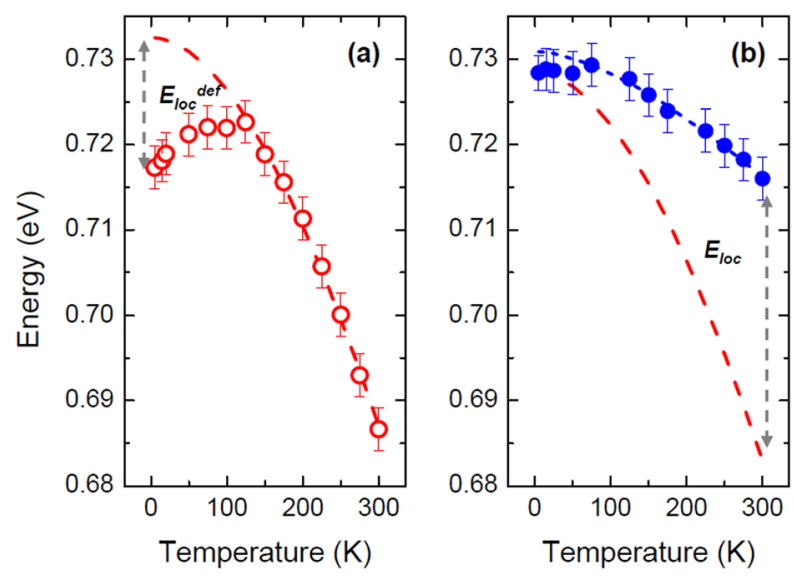

FIG. 3. Temperature dependence of the PL peak energy from (a) the InN film and (b) the MQW structure. Dashed lines indicate the theoretical evolution of the bandgap with temperature following Varshni equation, using the parameters reported in Ref. 10. 
equation points out a carrier localization energy $\mathrm{E}_{l o c}$ above $35 \mathrm{meV}$. The potential fluctuations responsible for this localization can be related to variations in the QW thickness (a fluctuation of \pm 2 ML leads to a change in the PL energy of $\pm 20 \mathrm{meV}$ ) or to alloy fluctuations in the InGaN barriers, which induce a certain degree of lateral confinement, enhanced by the intense piezoelectric field in InGaN alloys. ${ }^{15}$ Indeed, looking at the band diagram in Fig. 2(b), the internal electric field in the QWs is not fully screened for a residual doping of $10^{19} \mathrm{~cm}^{-3}$, although theoretical calculations (not shown) predict a decrease by almost a factor of 2 when increasing the doping level from $10^{19} \mathrm{~cm}^{-3}$ to $10^{20} \mathrm{~cm}^{-3}$.

The PL emission linewidth of the InN film follows the predicted broadening with temperature of $k_{B} T$. This relationship is followed by the emission of the MQW sample for temperatures below $200 \mathrm{~K}$. For higher temperatures, the FWHM strongly increases up to $\sim 138 \mathrm{meV}$ at RT. This behaviour can be explained by assuming two very close distributions of potential minima, whose emission merges into a single one for temperatures above $200 \mathrm{~K}$.

The interband relaxation time of the InN and MQW samples was measured using the degenerate pump-probe technique at RT. The pump and probe pulses were produced by a femtosecond laser source emitting at $1.55 \mu \mathrm{m}$ (repetition rate $=100 \mathrm{MHz}$, pulse width $=100 \mathrm{fs}$ ). The peak intensity was $0.35 \mathrm{GW} / \mathrm{cm}^{2}$ for the pump and 10 times weaker for the probe. More details about the setup can be found in Ref. 16 . Figure 4 shows the detected differential probe signal versus the pump-probe time delay for both samples. For the InN sample, the measured decay cannot be well fitted with a single-exponential function, as already observed by other authors, ${ }^{17-19}$ revealing the coexistence of various carrier relaxation paths. In order to investigate the origin of these different processes, power-dependent measurements were performed, showing that the initial relaxation time constant decreases from 30 to $13 \mathrm{ps}$ when the excitation carrier density increases from $n_{\text {exc }} \approx 4.3 \times 10^{18}$ to $3.8 \times 10^{19} \mathrm{~cm}^{-3}$ (inset of

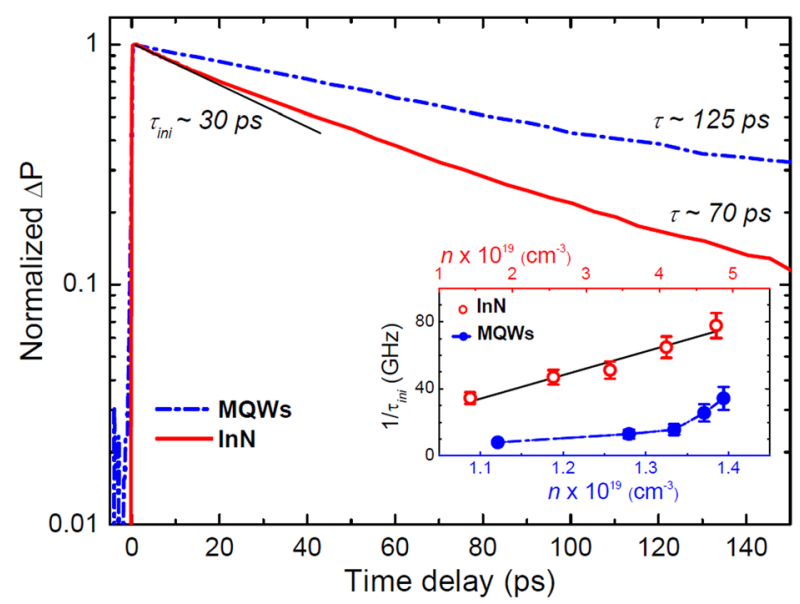

FIG. 4. Normalized differential detected probe signal as a function of the pump-probe time delay in both InN and MQW samples for a pump peak intensity of $0.35 \mathrm{GW} / \mathrm{cm}^{2}$. Inset: Dependence of the initial relaxation rate $1 / \tau_{\text {ini }}$ on the total electron density, which includes contributions from both background doping $\left(n_{0}\right)$ and photogeneration $\left(n_{\text {exc }}\right)$. The values of $n_{\text {exc }}$ are calculated taking into account the absorption coefficient and saturation intensity of the samples.
Fig. 4), which is in the same order that the residual carrier concentration of the layers, $n_{0} \approx 10^{19} \mathrm{~cm}^{-3}$. Since the role of radiative recombination is considered to be negligible in the picosecond scale, ${ }^{2}$ the observed relaxation should be dominated by a nonradiative recombination process, such as Auger. In the case of a degenerate system under high excitation $\left(n_{\text {exc }} \geq n_{0}\right)$, the Auger recombination rate $\left(1 / \tau_{\text {Auger }}\right)$ follows a linear dependence on carrier density $(n) .{ }^{20}$ The solid line in the inset of Fig. 4 is the linear fit to $1 / \tau_{\text {ini }}=A_{\text {defect }}$ $+C_{\text {Auger }} \cdot n$, where $A_{\text {defect }} \sim(14 \pm 5) \times 10^{-9} \mathrm{~s}^{-1}$ is the defect-related non-radiative recombination rate and $C_{\text {Auger }}$ $\sim(1.3 \pm 0.2) \times 10^{-9} \mathrm{~cm}^{3} \mathrm{~s}^{-1}$ is the Auger coefficient. The value of $C_{\text {Auger }}$ is slightly higher than the one obtained by Tsai et al. of $2.5 \times 10^{-10} \mathrm{~cm}^{3} \mathrm{~s}^{-1}$ in InN films grown by MBE on $\operatorname{Si}(111)^{17}$ pumping at $780 \mathrm{~nm}(1.59 \mathrm{eV})$. The second nonradiative relaxation mechanism, associated to the slower decay occurring at longer delays $(\tau \sim 70 \pm 7 \mathrm{ps}$, consistent with the obtained value of $A_{\text {defect }}$ ), is attributed to the recombination of the carriers via defects in the structure.

Regarding the MQW structure, a single-exponential decay with a longer defect-related carrier relaxation time $\tau \sim 125 \pm 10$ ps is obtained (Fig. 4) for low pump peak intensities $\left(0.35 \mathrm{GW} / \mathrm{cm}^{2}\right)$. However, when increasing the pump intensity, and thus the photogenerated carrier concentration, the decay becomes non-exponential. An estimation of the initial relaxation rate $\left(1 / \tau_{i n i}\right)$ as a function of the total electron density is depicted in the inset of Fig. 4. The change in the slope of $1 / \tau_{i n i}$ vs the total electron density is assigned to the activation of many-body phenomena.

In summary, the thermal behaviour of the PL emission from $\mathrm{InN} / \mathrm{In}_{0.9} \mathrm{Ga}_{0.1} \mathrm{~N}$ MQWs was analyzed and compared with a $1-\mu \mathrm{m}$-thick InN layer. The S-shape described by the PL energy peak as a function of the temperature in the $\mathrm{InN}$ film indicates carrier thermal delocalization from local potential minima related to structural defects, with an average localization energy of $12 \mathrm{meV}$. In the case of the MQWs, the localization energy increases above $35 \mathrm{meV}$, being attributed to potential fluctuations in the QWs due to layer thickness and/or alloy fluctuations. At the same time, longer carrier relaxation time $\sim 125 \mathrm{ps}$ is obtained from pump-probe measurements at $1.55 \mu \mathrm{m}$ for the MQW sample, as expected from the strong carrier localization present in the structure. This enhanced localization leads to an increase of the luminescence efficiency in the InN/InGaN MQW up to five times compared to the one obtained for bulk InN.

Partial financial support was provided by Spanish Government Project TEC2009-14423-C02-02, Comunidad de Madrid Project S2009/ESP-178, EC FP7 FET-OPEN Project Unitride under Contract No. 233950, and CEA-DSM-Energie NANIPHO Project.

${ }^{1}$ J. Wu, J. Appl. Phys. 106, 011101 (2009).

${ }^{2}$ F. B. Naranjo, M. González-Herráez, H. Fernández, J. Solís, and E. Monroy, Appl. Phys. Lett. 90, 091903 (2007).

${ }^{3}$ T. Ohashi, P. Holmstrom, A. Kikuchi, and K. Kishino, Appl. Phys. Lett. 89, 041907 (2006).

${ }^{4}$ S.-B. Che, T. Mizuno, X. Wang, Y. Ishitani, and A. Yoshikawa, J. Appl. Phys. 102, 083539 (2007).

${ }^{5}$ J. Grandal, J. Pereiro, A. Bengoechea-Encabo, S. Fernández-Garrido, M. A. Sánchez-García, E. Muñoz, E. Calleja, E. Luna, and A. Trampert, Appl. Phys. Lett. 98, 061901 (2011). 
${ }^{6}$ W. C. Ke, C. P. Fu, C. Y. Chen, L. Lee, C. S. Ku, W. C. Chou, W. H. Chang, M. C. Lee, W. K. Chen, W. J. Lin, and Y. C. Cheng, Appl. Phys. Lett. 88, 191913 (2006).

${ }^{7}$ M. Kumar, B. Roul, T. N. Bhat, M. K. Rajpalke, N. Sinha, A. T. Kalghatgi, and S. B. Krupanidhi, Adv. Sci. Lett. 3, 379 (2010).

${ }^{8}$ F. B. Naranjo, P. K. Kandaswamy, S. Valdueza-Felip, V. Calvo, M. González-Herráez, S. Martín-López, P. Corredera, J. A. Méndez, G. R. Mutta, B. Lacroix, P. Ruterana, and E. Monroy, Appl. Phys. Lett. 98, 031902 (2011).

${ }^{9}$ E. Bellet-Amalric, C. Adelmann, E. Sarigiannidou, J. L. Rouvière, G. Feuillet, E. Monroy, and B. Daudin, J. Appl. Phys. 95, 1127 (2004).

${ }^{10}$ S. Valdueza-Felip, L. Rigutti, F. B. Naranjo, B. Lacroix, S. Fernández, P. Ruterana, F. H. Julien, M. González-Herráez, and E. Monroy, Phys. Status Solidi A 209, 17 (2011).

${ }^{11}$ V. Potin, P. Ruterana, and G. Nouet, J. Phys.: Condens. Matter 12, 10301 (2000).

${ }^{12}$ Ž. Gačević, A. Das, J. Teubert, Y. Kotsar, P. K. Kandaswamy, Th. Kehagias, T. Koukoula, Ph. Komninou, and E. Monroy, J. Appl. Phys. 109, 103501 (2011).
${ }^{13}$ E. Monroy, N. Gogneau, F. Enjalbert, F. Fossard, D. Jalabert, E. Bellet-Amalric, L. S. Dang, and B. Daudin, J. Appl. Phys. 94, 3121 (2003).

${ }^{14}$ B. Liu, Z. Zhang, R. Zhang, D. Y. Fu, Z. L. Xie, H. Lu, W. J. Schaff, L. H. Song, Y. C. Cui, X. M. Hua, P. Han, Y. D. Zheng, Y. H. Chen, and Z. G. Wang, Appl. Phys. A 99, 139 (2010).

${ }^{15}$ M. Gallart, P. Lefebvre, A. Morel, T. Taliercio, B. Gil, J. Allègre, H. Mathieu, B. Damilano, N. Grandjean, and J. Massies, Phys. Status Solidi A 183, 61 (2001).

${ }^{16}$ L. Nevou, J. Mangeney, M. Tchernycheva, F. H. Julien, F. Guillot, and E. Monroy, Appl. Phys. Lett. 94, 132104 (2009).

${ }^{17}$ T.-R. Tsai, C.-F. Chang, and S. Gwo, Appl. Phys. Lett. 90, 252111 (2007).

${ }^{18}$ Y.-E. Su, Y.-C. Wen, H.-M. Lee, S. Gwo, and C.-K. Sun, Appl. Phys. Lett. 96, 052108 (2010).

${ }^{19}$ H. Ahn, K.-J. Yu, Y.-L. Hong, and S. Gwo, Appl. Phys. Lett. 97, 062110 (2010).

${ }^{20}$ A. Haug, Solid-State Electron. 21, 1281 (1978). 
Applied Physics Letters is copyrighted by the American Institute of Physics (AIP). Redistribution of journal material is subject to the AIP online journal license and/or AIP copyright. For more information, see http://ojps.aip.org/aplo/aplcr.jsp 